\title{
Concepção de Profissionais da Estratégia Saúde da Família sobre a Saúde Mental na Atenção Básica1
}

\author{
Denilson Gomes Silva² \\ José Nazareno Pearce de Oliveira Brito ${ }^{3}$ \\ Márcia Astrês Fernandes ${ }^{4}$ \\ Camila Aparecida Pinheiro Landim Almeida ${ }^{5}$ \\ Eliana Campêlo Lago ${ }^{3}$
}

\begin{abstract}
Este artigo teve como objetivo analisar a concepção de profissionais da Estratégia Saúde da Família sobre saúde mental com base na Política Nacional de Saúde Mental. Realizou-se uma pesquisa de campo, com abordagem qualitativa e a participação de 20 profissionais. Os dados foram coletados por meio de uma entrevista semiestruturada e investigados a partir da análise de conteúdo e agrupados em categorias. Foi possível identificar que a demanda relativa à saúde mental mais frequente é a de renovação de receitas médicas e as intervenções são fragmentadas por especialidades e encaminhamentos. Considerou-se a importância do apoio matricial envolvido com a responsabilização dos profissionais de saúde na intersetorialidade e integração das ações multidisciplinares de saúde mental com os usuários, família e comunidade.
\end{abstract}

Descritores: Profissionais de Saúde; Estratégia Saúde da Família; Saúde Mental.

\footnotetext{
${ }^{1}$ Artigo extraído de Dissertação de Mestrado "Concepção de Profissionais da Estratégia Saúde da Família sobre a Saúde Mental na Atenção Básica" apresentada ao Centro Universitário Uninovafapi, Teresina, PI, Brasil.

${ }^{2}$ Mestrando, Centro Universitário Uninovafapi, Teresina, PI, Brasil. Professor Titular, Instituto Superior de Teologia Aplicada, Sobral, CE, Brasil.

${ }^{3} \mathrm{PhD}$, Professor Titular, Centro Universitário Uninovafapi, Teresina, PI, Brasil.

${ }^{4} \mathrm{PhD}$, Professor Titular, Universidade Federal do Piauí, Teresina, PI, Brasil.

${ }^{5} \mathrm{PhD}$, Enfermeiro, Centro Universitário Uninovafapi, Teresina, PI, Brasil.
} 


\title{
Conception of Family Health Strategy Professionals on Mental Health in Primary Care
}

This article aims to analyze the design professionals of the Family Health Strategy on mental health based on the National Mental Health Policy. We conducted a field research with qualitative approach and the participation of 20 professionals. Data were collected through a semi-structured interview and investigated from the content analysis and grouped into categories. It was possible to identify the relative demand for more frequent mental health is the renewal of prescriptions medicals and interventions are fragmented by specialties and referrals. It considered the importance of matric support involved with the accountability of health professionals in intersectoriality and integration of actions multidisciplinarys to mental health with users, family and community.

Descriptors: Health Professionals; Family Health Strategy; Mental Health.

\section{Estrategia de Salud de los Profesionales de Diseño de la Familia de la Salud Mental en Atención Primaria}

\begin{abstract}
Este artículo tiene como objetivo analizar los profesionales del diseño de la Estrategia Salud de la Familia en la salud mental basado en la Política Nacional de Salud Mental. Se realizó una investigación de campo con enfoque cualitativo y la participación de 20 profesionales. Los datos fueron recolectados a través de una entrevista semiestructurada e investigados a partir del análisis de contenido y se agrupan en categorías. Fue posible identificar la demanda relativa de salud mental más frecuente es la renovación de las recetas médicas y las intervenciones fragmentado por especialidades y referencias. Se considera la importancia del apoyo matricial con la responsabilidad de los profesionales de la salud en enfoque intersectorial y la integración de acciones de salud mental multidisciplinar con los usuarios, familia y comunidad.
\end{abstract}

Descriptores: Profesionales de la Salud; Estrategia de Salud Familiar; Salud Mental.

\section{Introdução}

A Organização Mundial da Saúde (OMS), nos anos de 1970 a 1980, reconhece a magnitude dos problemas de saúde mental e admite a impossibilidade do seu cuidado ficar a cargo exclusivo de especialistas. Preconiza então a descentralização dos serviços existentes, a integração de serviços psiquiátricos em unidades de cuidados gerais, a formação de cuidadores não especializados e o aumento da participação da comunidade ${ }^{(1)}$.

Um dos desafios que se impõem ao Sistema Único de Saúde (SUS) na atualidade é a efetiva implementação da Reforma Psiquiátrica, consolidada com a Lei $n^{\circ}$. 10.216/2001. Ocorre que a Reforma Psiquiátrica garante a atenção integral à saúde aos usuários de serviços de saúde mental por meio da estruturação de serviços de base comunitária que devem ser configurados em redes assistenciais capazes de - em consonância com os princípios da Reforma Sanitária - observarem a equidade no atendimento e promover a reinserção social(2).

A integração da Estratégia Saúde da Família (ESF) com as políticas de saúde mental, por sua vez, implica também transformações profundas nas práticas de saúde institucionalizadas. A lógica dos "especialismos", ainda muito arraigada à cultura médica e hospitalocêntrica, encontrada entre os técnicos e mesmo entre a população 
usuária, dificulta a implementação de novas formas de cuidado. Tal lógica pressupõe relações hierarquizadas de saberes e poderes entre os diferentes membros da equipe e desta com os usuários ${ }^{(3)}$. Isso quer dizer que é preciso pôr em curso alterações na forma de organização dos serviços, pautadas por mudanças nos saberes instituídos que delimitam quem é competente e quem tem autoridade para lidar com o transtorno mental.

Os dados que permitam conhecer o nível de implementação de ações de saúde mental na rede básica ainda são muito incipientes. Buscando contribuir para a redução dessa lacuna, algumas pesquisas apresentam estudos de convergências com os princípios da Reforma Psiquiátrica, pois orientam-se com base em fundamentos epistemológicos que se situam em ruptura ao paradigma médico naturalista e clássico, atribuindo um novo lugar social para a loucura e, para isso, instaurando um Modelo Psicossocial de Cuidado ${ }^{(4)}$.

Este novo modelo deve situar-se em contradição com o modelo antigo, asilar, em quatro parâmetros principais: na definição de "seu objeto" e dos "meios" teóricos e técnicos de intervenção, nas formas da organização dos dispositivos institucionais, nas modalidades do relacionamento com os usuários e a população e nas implicações éticas dos efeitos de suas práticas em termos jurídicos, teóricos, técnicos e ideológicos ${ }^{(5)}$.

Valendo-se desses quatro parâmetros, o Modelo Psicossocial propõe que fatores políticos, biopsíquicos e socioculturais sejam tomados como determinantes das doenças ${ }^{(5)}$. E as terapias sairiam do escopo medicamentoso exclusivo, ou preponderante, e o sujeito ganharia destaque como participante principal no tratamento, sendo a família e, eventualmente, um grupo mais ampliado também incluídos como agentes fundamentais do cuidado.

Porém, destaca-se que no cenário da atenção básica, a maioria dos pacientes com transtorno mental não tem seu diagnóstico reconhecido e por isto não são devidamente tratados. Entre os principais motivos para o não reconhecimento dos transtornos mentais está a maior probabilidade destes pacientes relatarem apenas sintomas somáticos quando em consulta, e a dificuldade dos profissionais da ESF em reconhecer estes sintomas como indicativos de transtorno mental ${ }^{(5)}$.

A partir dessas informações, as práticas em saúde mental existentes no nível básico de atenção à saúde nem sempre condizem com o esperado e, assim, definiu-se como questão norteadora deste estudo: qual a concepção dos profissionais da Estratégia Saúde da Família sobre a saúde mental na atenção básica?

Para uma maior aproximação com a problemática apresentada e considerando as diretrizes da Política
Nacional de Saúde Mental, o cuidado em saúde mental não é algo de outro mundo ou para além do trabalho cotidiano na atenção básica. Pelo contrário, as intervenções são concebidas na realidade diária do território, com as singularidades dos pacientes e de suas comunidades ${ }^{(6)}$.

Diante do exposto, este estudo teve como objetivo analisar a concepção de profissionais da Estratégia Saúde da Família sobre a saúde mental com base na Política Nacional de Saúde Mental.

\section{Materiais e Métodos}

O presente estudo trata-se de uma pesquisa de campo, descritiva, exploratória e com abordagem qualitativa, pois proporciona uma compreensão mais abrangente da história, das representações, das crenças e relações dos participantes envolvidos no estudo ${ }^{(7)}$. A referida abordagem é coerente e, ao mesmo tempo, consistente ao tratar da subjetividade dos participantes do estudo, dentro do aporte metodológico de construção, análise e discussão dos dados, bem como respeito aos princípios éticos ${ }^{(8)}$

A pesquisa realizou-se em um Centro de Saúde da Família (CSF) no estado do Ceará. Os participantes da pesquisa foram 20 profissionais da ESF (05 enfermeiras; 02 médicas; 02 odontólogos; 08 agentes comunitários de saúde; e 03 técnicas de enfermagem).

Os critérios de inclusão foram os profissionais da ESF registrados no Cadastro Nacional de Estabelecimentos de Saúde (CNES) e com mais de 02 (dois) anos de experiência profissional no CSF. Foram excluídos do estudo os preceptores, estagiários e profissionais de outros serviços de assistência à saúde.

Os dados foram coletados por meio da técnica de entrevista com o roteiro semiestruturado detalhado e organizado, e com as seguintes perguntas abertas: Quais as demandas em saúde mental que são acompanhadas pelos profissionais da ESF e descrever as ações de saúde mental realizadas no CSF.

As entrevistas sucederam-se de forma espontânea, com duração média de 30 minutos e executadas nos meses de julho e agosto de 2015 nas dependências do cenário de estudo, conforme a disponibilidade dos participantes. Foram gravadas em áudio e transcritas na íntegra, após a aceitação explícita em participarem da pesquisa e da explicação detalhada do objetivo do estudo, e que assinassem o Termo de Consentimento Livre e Esclarecido (TCLE). Estabeleceu-se o anonimato, identificando-os por números naturais.

Para a análise das informações obtidas nas entrevistas, foi utilizada a técnica de análise categorial temática, que se organiza, operacionalmente, em três 
fases: pré-análise, exploração do material e tratamento dos resultados obtidos e interpretação(9). Desta forma, as entrevistas foram transcritas na íntegra; posteriormente os dados foram sistematizados de modo a manter os conteúdos básicos dos pensamentos e os significados das mensagens apresentadas pelos participantes. Vale informar que os temas surgidos dos relatos transcritos foram agrupados em unidades de contexto, formando três categorias temáticas em discussão com a literatura.

O projeto desta pesquisa seguiu os preceitos éticos e legais da Resolução 466/12 do Conselho Nacional de Saúde (CNS) e foi aprovado pelo Comitê de Ética em Pesquisa (CEP) do Centro Universitário UNINOVAFAPI em Teresina - Piauí, mediante o parecer consubstanciado $\mathrm{n}^{\circ} .: 1.415 .624$ e Certificado de Apresentação para Apreciação Ética - CAAE n.: 44611415.7.0000.5210.

\section{Resultados e Discussão}

Para efeito de estudo, obteve-se a participação de 20 profissionais da Estratégia Saúde da Família (05 enfermeiras; 02 médicas; 02 odontólogos; 08 agentes comunitários de saúde e 03 técnicas de enfermagem), com idade entre 30 a 50 anos e atuação profissional há mais de 02 anos no referido local da pesquisa.

E para a análise dos resultados, pode-se compilar em três categorias: o tipo de demanda em saúde mental vivenciada no Centro de Saúde da Família; as ações em saúde mental desenvolvidas e a importância do apoio matricial para os profissionais da Estratégia Saúde da Família. Segue a apresentação destas categorias e suas respectivas discussões:

\section{O tipo de demanda em saúde mental vivenciada no Centro de Saúde da Família}

De um modo geral, a demanda relativa à saúde mental mais frequente na realidade do Centro de Saúde da Família é a de pacientes que procuram o atendimento para renovar a receita médica e continuar o tratamento medicamentoso. Outras demandas também foram mencionadas, como o uso de álcool e outras drogas e situações constatadas como sendo de transtorno mental na área de cobertura da equipe. Contudo, a demanda pela renovação de receitas médicas parece ser mais comum e mais alarmante.

Vale destacar abaixo as verbalizações dos participantes:

Aparece demanda, paciente com queixa depressiva como hoje apareceu uma, que sofre angústia por perda de algum parente, paciente que faz uso crônico de medicação controlada (Participante 1).
A gente tem muitos pacientes que já vem usando medicamento há um tempo prescrito por um psiquiatra (Participante 3).

Aqui no CSF é atendida a mãe que faz uso de medicação, pois o sofrimento é o desaparecimento de um filho envolvido com drogas; mulher com câncer de mama fragilizada pelo tratamento; mulher com medo à noite; caso de maus tratos pelo marido (Participante 5).

Vem para o atendimento o homem usuário de drogas e que "ouve vozes" e quer tomar medicação psicotrópica; demanda de receita porque os remédios não estão funcionando bem e quer outro remédio mais forte (Participante 10).

Aqui sempre tem paciente da saúde mental. Eles já procuram a farmácia e querem o medicamento, já vem com a receita do psiquiatra e depois vão embora. A realidade infelizmente é esta: Eles querem somente receber as caixas de medicamentos (Participante 19).

Tentativa de suicídio por ingestão de diazepan, haloperidol ou tryptanol; homem que guarda potes de urina e lixo no quarto e quer tomar medicação para controlar as compulsões (Participante 11).

Tem demanda de laudo de psiquiatra do SUS para aposentar; vítima de estupro que tem crise de pânico e quer a medicação controlada; família que recorre ao CSF porque acha que a mãe está "doida" e precisa da medicação (Participante 17).

O modo que prevalece no acolhimento das demandas dos usuários com transtorno mental e também com conflitos familiares, comportamentos suicidas e em situação de drogadição e diversas violências, que foi expresso nas entrevistas indica o privilegiamento dos cuidados medicamentosos e especializados para a resolução dos problemas de saúde na população. Isto se reflete de duas maneiras: o encaminhamento dessas demandas para os serviços/profissionais especializados em saúde mental e a prescrição de receitas médicas.

O medicamento é visto como a principal prática terapêutica na rede pública de saúde, sendo muitas vezes considerado o único recurso disponível para aqueles que necessitam desse tipo de cuidado ou estão em situação de risco, tornando os usuários dependentes $^{(10)}$. Esse fenômeno está relacionado ao papel dominante da indústria farmacêutica na atenção à saúde da família e à força do modelo biomédico, ainda hegemônico no acompanhamento das queixas físicas, emocionais e sociais da população.

O processo da medicalização está muito presente nos dias de hoje e ocupa um lugar importante no jogo de interesses do poder econômico. O consumo de medicamentos tem um significativo impacto na sociedade, haja vista constituir o principal meio de combate às doenças e outras problemáticas relacionadas na prática terapêutica atual e também por ter relevante 
significância em termos econômicos. Aliado a isso, existe o fato de as consultas médicas resultarem quase sempre numa prescrição, decorrente de uma visão limitada da saúde, para a qual o medicamento tornouse a principal ferramenta ${ }^{(11)}$.

Por conseguinte, deixa-se de ter uma visão ampla das dificuldades e/ou vulnerabilidades dos usuários e seus determinantes, visto que a Estratégia Saúde da Família tem como principal proposta à reorganização da atenção em saúde para o desenvolvimento de atendimentos promovidos por uma equipe interdisciplinar orientada por concepções ampliadas do processo saúde/doença e pelo desenvolvimento de práticas psicossociais de cuidado centradas na família e compreendidas em seu território(3).

Para corroborar com as informações supracitadas, a atenção à saúde mental reorienta-se do modelo hospitalocêntrico para o de base comunitária. A incorporação de ações de saúde mental na atenção básica contribuirá para esta transformação, oferecendo maior cobertura assistencial e potencial de reabilitação psicossocial ${ }^{(12)}$. Mas, para isto, é importante entender que a execução dessas ações de saúde mental requer atuação marcante quanto à responsabilidade sanitária no território e resolutividade frente às questões apresentadas.

\section{As ações em saúde mental desenvolvidas no Centro de Saúde da Família}

Diante das demandas apresentadas no Centro de Saúde da Família, os profissionais de saúde, em geral, renovam as receitas psiquiátricas sem maiores questionamentos sobre essa estratégia de intervenção. É possível perceber que a concepção de ações em saúde mental está bastante compartimentalizada, com intervenções fragmentadas por especialidades.

Sendo assim, os entrevistados apresentaram estas respostas:

A gente recebe, mas geralmente se resume a paciente que já é acompanhado por um psiquiatra e vem apenas para a gente transcrever a receita (Participante 2).

Nós não temos condições de dar um suporte terapêutico, diagnóstico em saúde mental, que é uma coisa muito especializada (Participante 4).

Eu tenho várias dificuldades em desenvolver ações de saúde mental, por causa da falta de conhecimentos específicos em saúde mental, cuja nossa formação é generalista ou com especialidades em outras áreas. E assim, torna-se frágil minha participação em relação ao cotidiano das demandas de saúde mental (Participante 13).

As médicas do CSF geralmente encaminham para um especialista, porque elas não podem ficar prescrevendo esses medicamentos, né? Então tem que realmente encaminhar para um especialista. E aí elas têm feito isso (Participante 14).
Eu praticamente tenho que encaminhar para o psiquiatra, não me sinto totalmente segura para acompanhar esse tipo de paciente. Porém, eu tenho consciência do movimento da Reforma Sanitária e Psiquiátrica e o respaldo legal da Constituição e do SUS, que não é mais possível pensar na saúde de forma compartimentalizada, pois "mental" e "físico" não são dissociados (Participante 8).

Assim, eu penso que deveria ter a integralidade, mas na prática, cada profissional só faz sua especialidade e pronto. A atenção básica é responsável pela população de um território. Assim, não se pode deixar que seja responsável só por um aspecto da vida do ser humano, mas abranger tudo e ser efetivo de forma integral (Participante 12).

Tendo em vista esses relatos, percebe-se a crescente fragmentação pela qual a medicina e outras áreas da saúde passaram, levando à formação de áreas de conhecimentos distintas, com objetos de intervenção diferenciados e dificultando a produção de ações integrais em saúde mental que privilegiem a concretude da vida dos sujeitos.

A visão predominantemente fragmentada do trabalho em saúde mental, baseada nos conhecimentos especializados, propicia ainda o fortalecimento da lógica dos encaminhamentos ${ }^{(13)}$. Com isso, as intervenções em saúde mental pressupõem sempre uma lógica de hierarquização, havendo uma diferença de poder/saber entre quem encaminha e quem recebe e ainda uma transferência de responsabilidade.

A partir das entrevistas, observa-se que isso propicia e reforça a dificuldade desses profissionais diante das demandas em saúde mental apresentadas no CSF, além da cristalização das tradicionais intervenções em saúde mental, e na saúde global, de prescrição de medicamentos e encaminhamentos. Entretanto, muitas ações podem ser desenvolvidas em parceria com as equipes dos Centros de Atenção Psicossocial (CAPS's), tais como: consulta/atendimento, trabalhos em grupo, visita domiciliar, reuniões e capacitações com todos os profissionais envolvidos na atenção à saúde mental ${ }^{(14)}$

Dessa forma, para atender ao princípio da integralidade, é necessário incluir a atenção aos portadores de sofrimento psíquico nas ações básicas de saúde. A integralidade da assistência é entendida como conjunto articulado e contínuo de ações e serviços preventivos e curativos, individuais e coletivos, exigido para cada caso, em todos os níveis de complexidade ${ }^{(3)}$.

\section{A importância do apoio matricial para os profissionais da Estratégia Saúde da Família}

Em relação aos profissionais da Estratégia Saúde da Família, foi possível descrever que a proposta 
do apoio matricial ainda não está clara e há muitas incertezas e expectativas equivocadas por parte desses profissionais. As escassas definições identificaram a relevância do apoio matricial como uma estratégia de descentralização no atendimento em saúde mental e, assim, pode-se considerar os seguintes relatos:

Então na prática, com o apoio matricial, se objetiva que a gente lide melhor com essa demanda porque serviços especializados não dão conta, então eles querem que a ESF inclua um trabalho em saúde mental (Participante 18).

Tem que ocorrer de forma continua e sistemática o apoio matricial, pois não há articulação entre os serviços da rede básica e especializada, salvo quando se trata de alguns encaminhamentos, partindo deles para os serviços especializados. Quando acontece esse referenciamento não há acompanhamento do caso, nem retorno por parte do serviço especializado (Participante 6).

O apoio matricial é a necessidade de ter um suporte para o acolhimento em saúde mental (Participante 9).

A contribuição do apoio matricial às equipes poderia se realizar por meio da oferta de capacitações, treinamentos e palestras na área específica de saúde mental (Participante 7).

Eu acho que deve desenvolver o apoio matricial, porque é um canal que eles, os profissionais do CAPS têm para ouvir também a gente. Eles estão vendo que na prática não é tão fácil jogar a demanda de saúde mental para a equipe da ESF sem preparo, porque na prática a gente não vai ter capacitação para atender e o próprio doente não vai querer (Participante 20).

Para ter o apoio matricial, deve ter a presença de um psicólogo por Unidade Básica de Saúde, bem como de um veículo para o transporte de usuários com transtornos mentais (Participante 16).

Esse apoio matricial deveria ser um suporte emocional para os profissionais da ESF (Participante 15).

Concernente às respostas dos participantes, verifica-se que a perspectiva de um atendimento efetivo para a demanda em saúde mental na unidade básica é vislumbrada; entretanto, a possibilidade de um trabalho articulado entre a ESF e o serviço especializado não é percebida.

Uma proposta como essa, que envolve muitos atores em sua elaboração e execução, deve ser discutida por todos os envolvidos, visto que as ações deverão ser construídas em parceria no decorrer do processo. Não se trata, portanto, apenas de tomada de decisões no nível da gestão, de forma centralizada e isolada, mas de forma compartilhada e pactuada.

Tradicionalmente, o modelo hierarquizado de atendimento em saúde produziu fragmentação e burocratização nas formas de relacionamento entre os serviços de saúde, instituindo formas de se conceber e de se intervir nos equipamentos de saúde, repercutindo na desresponsabilização em vez de corresponsabilização entre profissionais e serviços ${ }^{(15)}$. Dessa forma, o cuidado com a demanda em saúde mental é visto como um acréscimo de trabalho e encarado como uma desresponsabilização dos serviços especializados.

Entretanto, a proposta do apoio matricial é articular a atenção básica e o serviço especializado, promovendo encontros de saberes que proporcionem uma atuação mais integral e menos fragmentada. Dessa forma, o CAPS, no papel de serviço especializado, não estaria desresponsabilizando-se de sua demanda, mas passando a atuar numa outra perspectiva, a de descentralizar esse cuidado, levando-o para mais perto do usuário(16). Os profissionais desse serviço estariam orientando e construindo, juntamente com os profissionais da atenção básica, um novo modelo de atenção, em que o maior beneficiado é o próprio usuário. Não há, portanto, um desvio e sim um compartilhamento de responsabilidades.

É um novo sistema de referência entre profissionais da ESF e usuários para a efetivação da proposta de apoio matricial, em que o paciente se utiliza de um serviço matricial e nunca deixa de ser cliente da equipe de referência. Neste sentido, não há encaminhamento, mas desenho de projetos terapêuticos que não são executados apenas pela equipe de referência, mas por um conjunto mais amplo de trabalhadores ${ }^{(17)}$. De qualquer forma, a responsabilidade principal pela condução do caso continua com a equipe da atenção básica.

Com isso, a proposta de apoio matricial faz-se a partir do movimento de ações integrativas e terapêuticas entre a equipe de referência da atenção básica e a equipe de outros serviços adequados à demanda de cada caso. Nesse sentido, os atendimentos em saúde mental serão acompanhados e fortalecidos por saberes específicos dos diversos profissionais de saúde.

Além disso, a aposta nesse arranjo está na troca de saberes entre equipes e profissionais em torno da busca de ofertas de ações/serviços que tenham potência para modificar positivamente os problemas de saúde (no seu amplo sentido) de um sujeito individual ou coletivo, com o uso, o mais racional possível, de toda ordem de recursos disponíveis ${ }^{(16)}$

É preciso o exercício cotidiano de cultivar uma relação respeitosa entre o saber generalista e o saber especializado, da mesma forma na relação entre o saber do usuário e do profissional de saúde. Será preciso considerá-los como legítimos saberes diferentes. É nesse sentido que o apoio matricial implica-se com uma lógica de troca multidirecional, de duplo ganho aos participantes do processo (aprender e ensinar), e não numa corrente hierarquizada e descendente que 
coloca os saberes e as práticas cotidianas dos serviços da atenção básica em lugar de subserviência à uma maneira naturalizada de considerar a competência do especialista.

\section{Considerações Finais}

Considera-se que os resultados da pesquisa apontaram a preeminência do paradigma biomédico na atenção à saúde, em que se privilegiam os cuidados medicamentosos na resolução dos problemas de saúde da população, como a prescrição indiscriminada de psicotrópicos. A visão predominantemente fragmentada do trabalho em saúde, por sua vez, ancorada nos conhecimentos especializados, propicia ainda a ampliação da proposta dos encaminhamentos.

Estes entraves foram identificados por meio das dificuldades dos profissionais da atenção básica em desenvolver uma concepção sobre as particularidades da saúde mental, advindas desde a formação, assim como a grande demanda da clientela pela qual se responsabilizam no trabalho, restando pouco tempo para a intersetorialidade e reabilitação psicossocial dos usuários com transtorno mental.

O estudo esclareceu que o envolvimento dos profissionais da atenção básica, por si só, não será suficiente para reorganizar as práticas no campo da atenção em saúde mental, pois as ações não se restringem exclusivamente ao setor saúde. Vale intensificar as políticas intersetoriais, indissociáveis, ampliando possibilidades e oportunidades, auxiliando um progresso local e redimensionando a noção de direito e cidadania. Isto remete à necessidade explicitada de construção de redes, de e entre serviços.

Portanto, torna-se premente trabalhar para a efetiva implementação da rede de cuidados em saúde mental na atenção básica, no sentido do trabalho interdisciplinar e a participação da comunidade, vinculado à percepção do processo saúde e doença nos componentes subjetivos, físicos e sociais dos usuários e familiares.

\section{Agradecimentos}

Aos professores do Programa de Mestrado Profissional em Saúde da Família do Centro Universitário - Uninovafapi.

\section{Referências}

1. Gonçalves AM, Sena RR. Psychiatric reform in Brazil: contextualization and consequences regarding the care for the mentally ill in their family environment. Rev. LatinoAm. Enfermagem. São Paulo. 2010;9(2):48-55.
2. Barroso SM, Silva MA. Reforma Psiquiátrica brasileira: o caminho da desinstitucionalização pelo olhar da historiografia. Rev. SPAGESP. São Paulo. 2011;12(1):66-78.

3. Mattos RA. A integralidade na prática (ou sobre a prática da integralidade). Cad. Saúde Pública. Rio de Janeiro. 2012;20(5):1411-16.

4. Figueiredo MD, Campos RO. Saúde mental na atenção básica à saúde de Campinas, SP: uma rede ou um emaranhado? Ciência e Saúde Coletiva. Rio de Janeiro. 2013;14(1):129-38.

5. Alverga A, Dimenstein M. Salud mental en la atención básica. Construyendo La integralidad en el Sistema Unico de Salud en Brasil. Revista Alternativas en Psicología. México. 2010;40(3):67-77.

6. Amaral MA. Atenção à saúde mental na rede básica: estudo sobre a eficácia do modelo assistencial. Rev. Saúde Pública. Rio de Janeiro. 2014;31(3):288-95.

7. Gil AC. Métodos e técnicas de Pesquisa Social. 6. ed. São Paulo: Atlas; 2011.

8. Bosi MLM. Pesquisa qualitativa em saúde coletiva. Ciência e Saúde Coletiva. Rio de Janeiro. 2012;17(3):575-86.

9. Bardin L. Análise de conteúdo. 70. ed. São Paulo; 2015. 10. Carvalho LF, Dimenstein M. O modelo de atenção à saúde e o uso de ansiolíticos entre mulheres. Estudos de Psicologia. Natal. 2011;9(1):121-29.

11. Souza AMA, Braga VAB. Brazilian psychiatric reform: a lot to reflect on. Acta Paul. Enferm. São Paulo. 2012;7(1):131-42.

12. Mostazo RR, Kirschbaum DIR. Users of a psychosocial care center: a study of their social outcomes about psychiatric treatment. Rev. Latino-Am. Enfermagem. São Paulo. 2013;11(6):786-91.

13. Souza LV, Santos MA. Group process and psychologist work in primary health care. Rev Bras Cresc e Desenv Humano. São Paulo. 2012;22(3):388-95.

14. Pitta AMF. Reorientação do modelo de atenção: equidade e justiça social na organização de serviços de saúde mental. Rev. SPAGESP. São Paulo. 2013;18(1):70-8. 15. Bower $P$, Sibbald $B$. On-site mental health workers in primary care: effects on professional practice. Acta Paul. Enferm. São Paulo. 2013;12(1):160-72.

16. Nicácio MFS. Utopia da realidade: contribuições da desinstitucionalização para a invenção de serviços de saúde mental. Arq. Ciênc. Saúde. São Paulo. 2014;15(2):140-52. 17. Yasui S. Rupturas e encontros: desafios da reforma psiquiátrica brasileira. Ciência e Saúde Coletiva. Rio de Janeiro. 2015;12(1):30-43. 